\title{
A Survey Paper on Fake News Detection Techniques
}

\author{
Vanita Babanne', Ashokkumar Thakur ${ }^{2}$, Sujit Shinde ${ }^{3}$, Tejas Patil ${ }^{4}$, Brijesh Gaud $^{5}$ \\ Department of Computer Engineering, RMDSSOE, Savitribai Phule Pune University, Pune, India ${ }^{1-5}$
}

\begin{abstract}
Fake news is counterfeit information which is mostly not true or layered. It has been around for a long time and with the coming of online life and cutting edge reporting at its pinnacle, the discovery of fake news has been a well-known point in the exploration network. There are various difficulties associated with the detection of deceptive news. This paper describes and compares various fake news detection systems, techniques and approaches and gives comparative analysis on them.
\end{abstract}

Keywords: Counterfeit, Pinnacle, Exploration, Deceptive

\section{INTRODUCTION}

Spreading of fake news has become center of attraction to various researchers as well as common people. Hence detection of fake news and its ceasing is the prime goal. The history is a witness that the impact of abundant of fake news on social media has led to mass lynching and riots. Hence in future, this scenario should not repeat, we need to focus on these challenge called as fake news proliferation. News comprises three things headlines, body, and images. Headlines can be falsely coated or fabricated even the images-can be photo shopped by hoaxes spreaders, leading to spreading of false information reaching to the masses. Most of the people get manipulated by reading the headlines or seeing the images. The body of the news is the main content of the news and most of the people don't read it and fake news spreaders take advantage of this scenario as discussed.

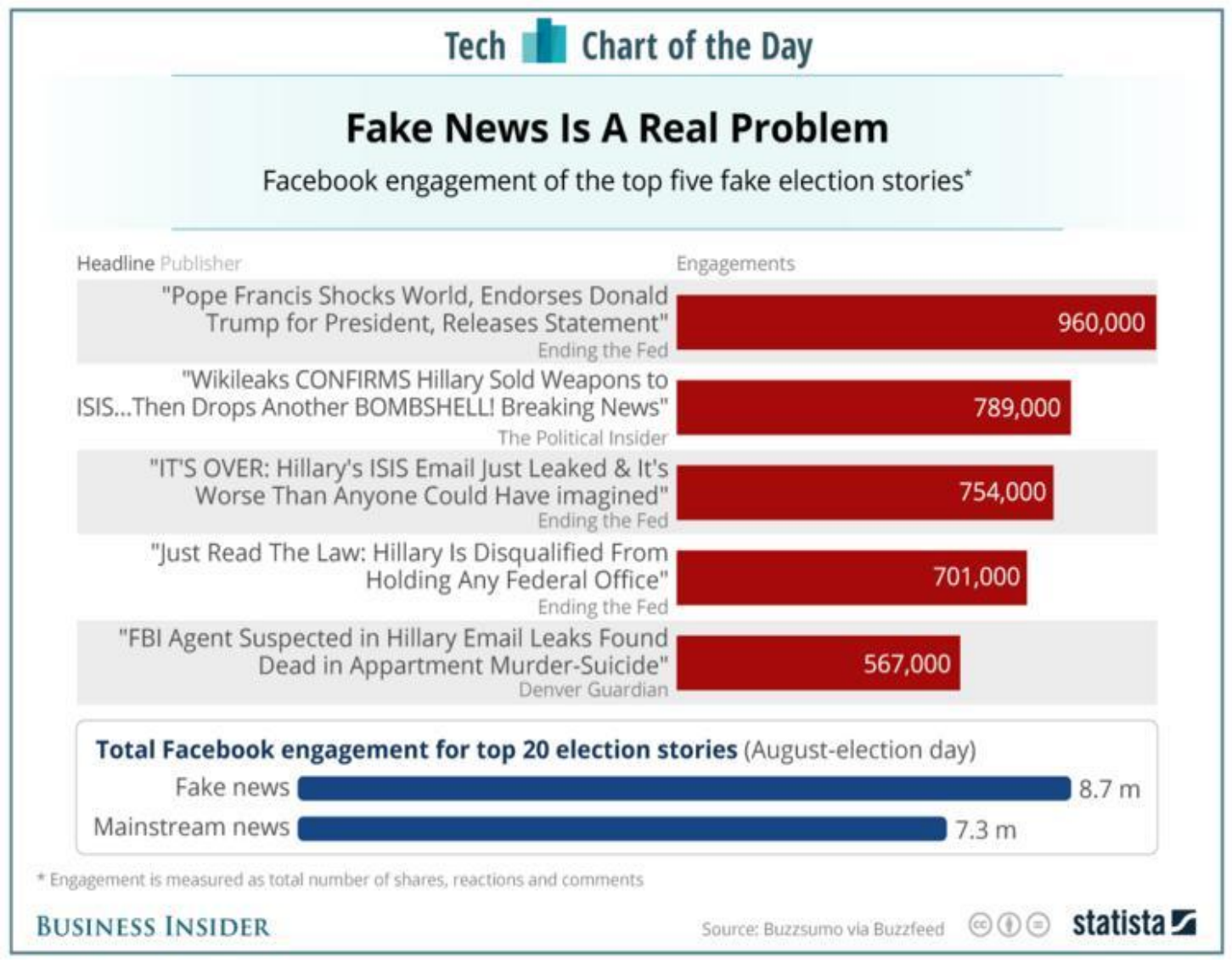

Fig. 1. Fake News In Trend 
Vol. 7, Issue 12, December 2018

Social media can reach out to millions of users within minutes. Fake news stories are very reachable and have a very high impact factor. Social media has become the main source of information for many people worldwide and because various organizations and people are trying to take advantage of this situation by tarnishing the image of an individual, organizations, societies. It is difficult to spot fake news on social media because there is no governance of publishing information and no control over spreading information, anyone can spread any information about anything or anyone at any time. This can lead to the creation of tension in various groups, cultures, societies and even the manipulation at individual level. For example, the U.S. presidential election, this led to the manipulation of the voters. The automatic fake news detection systems enable identification of deceptive news or misleading information. Low accuracy remains the main drawback of these systems. Even the system confuses with the satirical stories that are designed to entertain the reader by ridiculing or criticizing a social guru/organization. Another drawback is that large dataset for training and testing is not available and even if available then rich dataset is not available.

\section{TYPES OF FAKE NEWS}

$\bullet$

Visual based: - It has false, morphed or photo shopped images and even edited videos, which leads to fake

news.

- Satire or parody: - Its intention is just to entertain people and not spread misleading information but it has the ability to fool people. For example, fake tweets from famous personalities or memes.

- Different connection: - Here the headline or the images is contrasting or does not matches the body, this is usually done to mislead people because most of the people only read the headline or see the images.

- $\quad$ Style based copying:- Here fake news try to match the writing standards and writing styles of reputed authors. Leading to the spread of fake information so that people believe it has come from reputed sources.

\section{APPROACHES FOR FAKE NEWS DETECTION}

\section{Fake News Style Detection}

Fake news style detection is an approach to detect fake news by analyzing the style of the news. This approach assumes that the real news is well written, and based on this assumption it classifies the news. But it will not work on every case. Fake news can appear in well-written articles and vice-versa. Content of the news is more important than the style of news.

\section{Crowd Sourcing}

In this approach news that needs to verified is put on the platform and peoples votes for the news either true or fake. Based upon the votes news are classified.

\section{Social Argumentation by experts}

In this approach news are put on the platform but rather than taking votes from people experts opinions are taken and based upon the expert's opinions news are classified.

\section{Machine Learning Approach}

In this technique various machine learning algorithms are used to classify the news. Various models are made and trained by using the training data and then these models are tested on the testing data. Based upon its performance in training and testing phase accuracy is determined.

\section{LITERATURE SURVEY}

[1] Presented a technique to detect fake news using Naive Bayes classifier. The main idea is to treat each word of the news article independently. This technique checks the conditional probability of the facts, that news article is fake given that it contains some specified words. This approach was implemented as a software system and tested against a data set of Facebook news posts. This achieved classification accuracy of approximately $74 \%$ on the test set.

[2] Presented a model which is spurred by the three qualities of fake news for example the content of an article, the client reaction it gets, and the source client advancing it. In light of these three qualities display is made out of three modules: Capture, Score, and Integrate. Capture depends on the reaction and content. To remove transient portrayal of articles Recurrent Neural Network (RNN) is used. Score module learns the source qualities dependent on the conduct of clients, and these two modules incorporated with third module to group an article as fake or genuine. 
[3] Developed a method for fake news recognition on Twitter by figuring out how to anticipate exactness assessment in two believability centered Twitter dataset: CREDBANK and PHEME. CREDBANK is a publicly supported dataset of precision assessment for occasions in Twitter. PHEME is a dataset of potential talk in Twitter and journalistic evaluation of their exactness. This technique is then connected to Twitter content sourced from Buzz feed's fake news dataset. An element examination at that point identies highlights that are most prescient for publicly supported and journalistic precision evaluations, aftereffects of which are steady with earlier work.

[4] Proposed a novel ML fake news detection method which, by combining news content and social context features, outperforms existing methods in the literature, increasing their already high accuracy by up to $4.8 \%$. Second, they implemented method within a Facebook Messenger Chabot and validate it with a real-world application, obtaining a fake news detection accuracy of $81.7 \%$

[5] The authors describe a system in which they exploit the echo chamber communities i.e. the communities that share the same belief that exist in the social media. Because the spreading of fake news on echo chambers is easy for this they use 3-mode tensor representation of the news - <News, User, Community>. It is a novel state of art technique.

[6] The authors describe a system in which they use machine learning classification models combined with the proposed data preprocessing methods. They have used data imputation for handling of categorical and missing values. Used TF-IDF vectorization for feature extraction to lter out irrelevant features. Used Multi-Layer Perceptron (MLP) classier and the accuracy is increased by $15 \%$.

[7] Addresses the fake news and satire detection by proposing a method that uses a hybrid machine-crowd approach for detection of potentially deceptive news. This system com-bines the human factor with the machine learning approach and a decision-making model that estimates the classification confidence of algorithms and decides whether the task needs human input or not. This approach achieves reasonably higher accuracy compared other results, in exchange of cost and latency of using the crowdsourcing service. Logistic Regression (Log), SVM, Random Forest (RF), Neural Networks (NN), and Gradient Boosting Classifier (GDC) Machine learning classifications models are used. While crowdsourcing works on the judgments of online cloud works.

[8] Uses advance framework for fake news detection including techniques like statistical analysis of twitter user accounts, reverse image searching, cross verification of fake news sources and data mining. The framework contains two components core and website. Core is responsible for fetching the tweets content, constructing the feature set using the tweet dataset provided by the dataset, building the classifier and generating the evaluation report. website is to provide tweet credibility predictions, tweet details and crowd sourcing results.

[9] System is countering the spread of fake news on social networks by leveraging these crowds to instead help verify alternative facts. A prototype of social argumentation framework to verify the validity of proposed alternative facts to help curb the propagation of fake news. It utilizes fundamental argumentation ideas in a graph-theoretic framework that also incorporates semantic web and linked data principles. The argumentation structure is crowdsourced and mediated by expert moderators in a virtual community. It consists of three main components: The Graph-Theoretic Framework, the User Interface Component, and the Virtual Community to support crowdsourcing.

[10] There are three data sets of true and lying texts collected for the fake news detection, and automatic classification is a viable technique to distinguish between truth and falsehood as expressed in language. It also introduces a method for class-based feature analysis, which sheds some light on the features that are characteristic for deceptive text.

[11] The performance of detecting fake news only from content is not satisfactory, and it is suggested to incorporate user social engagements to improve fake news detection. It will construct a real-world datasets measuring users trust level on the fake news. There are two type of users" Experienced" users who can recognize fake news and" naive" who can believe fake news. A comparative analysis over implicit and explicit profile features between these users which reveals their potential to differentiate fake news.

[12] It uses a weekly supervised approach, which will collect a large amount of noisy dataset from many tweets. During collection of the data, tweets are classified to trustworthy and untrustworthy source and train classifier on these datasets. Then the system uses these classifiers for the detection of fake news i.e. fake tweets. To improve the classification of trustworthy and untrustworthy sources a factor F1 is used up to 0.9 . 


\section{IJARCCE}

Vol. 7, Issue 12, December 2018

\section{COMPARISON TABLE}

\begin{tabular}{|c|c|c|c|}
\hline Author & Year & Approach & Description \\
\hline $\begin{array}{l}\text { Mykhailo Granik and } \\
\text { Volodymyr Mesyura }\end{array}$ & 2017 & Naïve Bayes Classifier & $\begin{array}{l}\text { They determined conditional probability of } \\
\text { words to classify news }\end{array}$ \\
\hline $\begin{array}{l}\text { Natali } \quad \text { Ruchansky, } \\
\text { Sungyong Seo and Yan Liu }\end{array}$ & 2017 & Deep Learning & $\begin{array}{l}\text { They developed three modules Capture, } \\
\text { Score and Integrate and combine them } \\
\text { together for classification }\end{array}$ \\
\hline $\begin{array}{l}\text { Cody Buntain and Jennifer } \\
\text { Golbeck }\end{array}$ & 2017 & $\begin{array}{l}\text { Use of } \quad \text { two } \\
\text { believability centered } \\
\text { Twitter dataset }\end{array}$ & $\begin{array}{l}\text { They trained their model on two Twitter } \\
\text { dataset: CREDBANK and PHEME }\end{array}$ \\
\hline $\begin{array}{l}\text { Marco L. Della Vedova, } \\
\text { Eugenio Tacchini, Stefano } \\
\text { Moret and Gabriele } \\
\text { Ballarin }\end{array}$ & 2018 & $\begin{array}{l}\text { combination of news } \\
\text { content and social } \\
\text { context features }\end{array}$ & $\begin{array}{l}\text { Implemented method within a Facebook } \\
\text { Messenger Chabot and validate it with a } \\
\text { real-world application }\end{array}$ \\
\hline $\begin{array}{l}\text { Shashank Gupta, } \\
\text { Raghuveer Thirukovalluru, } \\
\text { Manjira Sinha and Sandya } \\
\text { Mannarswamy }\end{array}$ & 2018 & $\begin{array}{ll}3 \text {-mode } & \text { tensor } \\
\text { representation } & \end{array}$ & $\begin{array}{l}\text { Modelling of echo chambers for fake news } \\
\text { detection proposed Tensor Factorization } \\
\text { based method and its extension, a coupled } \\
\text { matrix-tensor factorization based method }\end{array}$ \\
\hline $\begin{array}{l}\text { Chandra Mouli Madhav } \\
\text { Kotteti, Xishuang Dong, Na } \\
\text { Li and Lijun Qian }\end{array}$ & 2018 & $\begin{array}{l}\text { Machine Learning } \\
\text { Classification }\end{array}$ & $\begin{array}{l}\text { Used Multi-Layer Perceptron for } \\
\text { classification and used data imputation for } \\
\text { data preprocessing thus increasing accuracy }\end{array}$ \\
\hline $\begin{array}{l}\text { Shaban Shabani and Maria } \\
\text { Sokhn }\end{array}$ & 2018 & $\begin{array}{l}\text { Combination r of } \\
\text { Crowd Sourcing and } \\
\text { Machine Learning } \\
\text { Techniques }\end{array}$ & $\begin{array}{l}\text { They combine human factor with the } \\
\text { machine learning approach and a decision- } \\
\text { making model }\end{array}$ \\
\hline $\begin{array}{l}\text { Saranya Krishnan and Min } \\
\text { Chen }\end{array}$ & 2018 & statistical analysis & $\begin{array}{l}\text { They uses techniques like statistical } \\
\text { analysis of twitter user accounts, reverse } \\
\text { image searching, cross verification of fake } \\
\text { news sources and data mining }\end{array}$ \\
\hline Ricky J. Sethi & 2017 & $\begin{array}{l}\text { Social Argumentation } \\
\text { Framework }\end{array}$ & $\begin{array}{l}\text { Argumentation structure is crowdsourced } \\
\text { and mediated by expert moderators in a } \\
\text { virtual community }\end{array}$ \\
\hline $\begin{array}{l}\text { Rada Mihalcea and Carlo } \\
\text { Strapparava }\end{array}$ & 2009 & $\begin{array}{ll}\text { class-based } & \text { feature } \\
\text { analysis } & \end{array}$ & $\begin{array}{l}\text { Automatic classification, technique to } \\
\text { distinguish between truth and falsehood as } \\
\text { expressed in language. }\end{array}$ \\
\hline $\begin{array}{l}\text { Kai Shu, Suhag Wang and } \\
\text { Huan Liu }\end{array}$ & 2018 & \begin{tabular}{llr}
\multicolumn{2}{l}{ Understanding } & User \\
Proles on & Social \\
Media & &
\end{tabular} & $\begin{array}{l}\text { They suggested to incorporate user social } \\
\text { engagements to improve fake news } \\
\text { detection. }\end{array}$ \\
\hline $\begin{array}{l}\text { Stefan Helmstetter and } \\
\text { Heiko Paulheim }\end{array}$ & 2018 & $\begin{array}{ll}\text { Weakly } & \text { Supervised } \\
\text { Learning } & \end{array}$ & $\begin{array}{l}\text { During collection of the data, tweets are } \\
\text { classified to trustworthy and untrustworthy } \\
\text { source and train classifier on these datasets. } \\
\text { Then the system uses these classifier for the } \\
\text { detection of fake news }\end{array}$ \\
\hline
\end{tabular}

\section{CONCLUSION}

In this paper, before coming up with new solutions, it is necessary to survey state of the art techniques for learning purposes for fake news detection. We covered multiple aspects of research problem of fake news detection, stated various fake news types. We surveyed various available techniques, compared various fake news detection systems, techniques and approaches and gave comparative analysis on them. 


\title{
IJARCCE
}

\section{International Journal of Advanced Research in Computer and Communication Engineering}

\author{
Vol. 7, Issue 12, December 2018
}

\section{REFERENCES}

[1]. Mykhailo Granik and Volodymyr Mesyura, "Fake News Detection Using Naive Bayes Classifier," 2017 IEEE First Ukraine Conference on Electrical and Computer Engineering (UKRCON).

[2]. Natali Ruchansky, Sungyong Seo and Yan Liu, "CSI: A Hybrid Deep Model for Fake News Detection, ”CIKM17, Singapore, Singapore.

[3]. Cody Buntain and Jennifer Golbeck, "Automatically Identifying Fake News in Popular Twitter Threads," 2017 IEEE International Conference on Smart Cloud.

[4]. Marco L. Della Vedova , Eugenio Tacchini, Stefano Moret and Gabriele Ballarin, “Automatic Online Fake News Detection Combining Content and Social Signals," PROCEEDING OF THE 22ND CONFERENCE OF FRUCT ASSOCIATION.

[5]. Shashank Gupta, Raghuveer Thirukovalluru, Manjira Sinha and Sandya Mannarswamy, "CIMTDetect: A Community Infused Matrix-Tensor Coupled Factorization Based Method for Fake News Detection,” 2018 IEEE/ACM International Conference on Advances in Social Networks Analysis and Mining (ASONAM).

[6]. Chandra Mouli Madhav Kotteti, Xishuang Dong, Na Li and Lijun Qian, "Fake News Detection Enhancement with Data Imputation," 2018 IEEE 16th Int. Conf. on Dependable, Autonomic Secure Comp., 16th Int. Conf. on Pervasive Intelligence Comp., 4th Int. Conf. on Big Data Intelligence Comp., and 3rd Cyber Sci. Tech. Cong.

[7]. Shaban Shabani and Maria Sokhn, "Hybrid Machine-Crowd Approach for Fake News Detection," 2018 IEEE 4th International Conference on Collaboration and Internet Computing.

[8]. Saranya Krishnan and Min Chen, "Identifying Tweets With Fake News," 2018 IEEE International Conference on Information Reuse and Integration for Data Science.

[9]. Ricky J. Sethi, "Spotting Fake News: A Social Argumentation Framework for Scrutinizing Alternative Facts," 2017 IEEE 24th International Conference on Web Services.

[10]. Rada Mihalcea and Carlo Strapparava, "The Lie Detector: Explorations in the Automatic Recognition of Deceptive Language," Proceedings of the ACL-IJCNLP 2009 Conference Short Papers.

[11]. Kai Shu, Suhag Wang and Huan Liu,"Understanding User Proles on Social Media for Fake News Detection," 2018 IEEE Conference on Multimedia Information Processing and Retrieval.

[12]. Stefan Helmstetter and Heiko Paulheim, "Weakly Supervised Learning for Fake News Detection on Twitter," 2018 IEEE/ACM International Conference on Advances in Social Networks Analysis and Mining (ASONAM).

[13]. https://en.wikipedia.org/wiki/Fake news.

[14]. https://libraryguides.vu.edu.au/c.php?g=460840\&p=5330649

[15]. https://www.businessinsider.in/Facebooks-fake-news-problem-in-onechart/articleshow/55504767.cms 